\title{
Foreword to the special issue on "Robustness, Knightian uncertainty, and games in finance"
}

\author{
Frank Riedel $^{1}$ - Chris Shannon ${ }^{2} \cdot$ Jan $_{\text {Werner }^{3}}$
}

Published online: 14 December 2017

(C) Springer-Verlag GmbH Germany, part of Springer Nature 2017

In recent years, the related issues of Knightian uncertainty and robustness have emerged as major themes in financial economics and decision theory. A rich theory of decision making under Knightian uncertainty has been developed in decision theory, complemented by a rich literature on risk measures in mathematics and finance. Both research strands emphasize the need to deal with foundational modeling assumptions. At the same time, due in part to the financial crisis starting in 2007, the need for "robust finance" became clear both in practice and in theory, and since then robust finance has become one of the main problems in the field.

In 2015, motivated by these problems, we had the pleasure to welcome an interdisciplinary group of researchers from economics, mathematical finance, and philosophy to form a research group at Bielefeld's Center for Interdisciplinary Studies, ZiF. The title of the 6month long workshop was "Robust Finance: Strategic Power, Knightian Uncertainty, and the Foundations of Economic Policy Advice". The research group worked on issues of robustness, Knightian uncertainty, and their implications for individual behavior, strategic interactions, and market outcomes. Thematic semesters and workshops on those and related themes have been held at other research centers and institutes following the $2015 \mathrm{ZiF}$ workshop.

The special issue of MAFE presents some outcomes of the research effort of the $\mathrm{ZiF}$ workshop. Workshop participants and other interested researchers were invited to submit papers on topics of robustness, Knightian uncertainty, and games in finance. We are happy to present this special volume to the readership of MAFE.

The contributions in this issue reflect the range of work in economics and finance related to robustness and Knightian uncertainty. This includes fundamental issues regarding the modeling and construction of nonlinear (backward) differential equations arising in the robust pricing of contingent claims and in dynamic recursive utilities under Knightian uncertainty,

\footnotetext{
Frank Riedel

frank.riedel@uni-bielefeld.de

1 Bielefeld University, Bielefeld, Germany

2 University of California, Berkeley, USA

3 University of Minnesota, Minneapolis, USA
} 
applications in investment and asset pricing, foundations for a class of measures of return risk, and modeling the perception of symmetry in the presence of Knightian uncertainty.

Christoph Belak, Thomas Seiferling, and Frank Seifried introduce the class of backward nonlinear expectation equations that arise in the robust pricing of contingent claims and in dynamic recursive utilities under Knightian uncertainty, both in discrete and continuous time. They generalize the ubiquitous backward stochastic differential equations to nonlinear expectations covering Shige Peng's notions of $g$ - and $G$-expectations as special cases. The authors show that the discrete time formulations of recursive utility in the spirit of Kreps and Porteus converge to their continuous-time counterparts; they thus provide, among other things, the rigorous foundation for Epstein and Ji's version of stochastic differential utility under Knightian uncertainty.

While the basic frictionless investment problems in continuous time are well understood, a lot of work remains to be done for complex decisions involving several underlying assets and frictions. Sören Christensen and Paavo Salminen provide a new approach to irreversible investment problems with more than one underlying asset. The basic literature on real options that started with the seminal contribution by McDonald and Siegel on the value of waiting to invest draws on an analogy to the pricing of American put options. Subsequently, a rich literature on investment under irreversible investment emerged. Sören Christensen and Paavo Salminen show that the Riesz representation of excessive functions provides a powerful tool to study optimal stopping problems. They show how the new approach can be successfully applied to the complex problem of "exchanging baskets". In that problem, an investor owns a portfolio of assets, modeled typically as geometric Brownian motions, that he can exchange for a new investment project with uncertain prospects. The exchange involves sunk costs for the new project and is thus irreversible. The aim is to find the optimal time to exchange the portfolio for the new project.

The paper by Klibanoff, Mukerji, and Seo, "Symmetry Axioms and Perceived Ambiguity", addresses a fundamental and elegant question: how is the perception of symmetry reflected in decision making under uncertainty and ambiguity? In a classic version of this problem, a decision maker faces uncertainty given by an infinite sequence of random variables. If the underlying random variables are actually independent and identically distributed (iid), such as repeated tosses of a given coin with unknown odds, and the decision maker knows the symmetry in the problem, so knows the process is exchangeable, this perception can be captured by a symmetry condition with respect to finite permutations, applied to preferences over acts based on outcomes of this sequence. One version of the classic de Finetti theorem then implies that a sharper expected utility representation obtains that reflects the decision maker's certainty that process is iid, and that there is no ambiguity in each random variable, with the only uncertainty the distribution of this random variable. Ambiguity is natural in this setting, for example reflecting the difference between repeatedly flipping a fixed coin and repeatedly drawing from an urn with a fixed number of balls of two colors but unknown distribution. Ambiguity could arise regarding realizations, or the relationship across random variables in the sequence, or combinations of the two, and modeling the perception of symmetry in this setting is subtle. A rich literature has considered this question, including proposing several notions of symmetry to generalize the classic exchangeability-based notion. In prior work, Klibanoff, Mukerji, and Seo provided an important contribution to this line by characterizing the class of preferences that can be represented by a monotonic aggregation of a family of subjective expected utilities generated by a von Neumann-Morgenstern utility for outcomes and a set of iid probability measures. The key preference condition underlying their results was a symmetry axiom, which they called event symmetry. In the current paper, their 
main result provides a unifying framework, showing that for a wide class of preferences many symmetry conditions proposed in the previous literature are equivalent to event symmetry.

The paper by Pennesi, "Asset Prices in an Ambiguous Economy," considers the asset pricing problem in a Lucas tree economy with a representative consumer. Starting with the seminal work of Epstein and Wang using recursive maxmin expected utility, prior work had characterized the equilibrium asset pricing implications of ambiguity using different specific forms of recursive ambiguity averse preferences for the investor. A key result in these papers is an analogue of the standard Euler equation characterization of equilibrium asset prices that obtains for an expected utility investor. One of the most celebrated findings in this literature was that in some cases, notably the maxmin expected utility model, the Euler equation becomes an Euler inequality, and ambiguity can thus lead to equilibrium price indeterminacy. Many authors interpret this as an equilibrium version of the classic result of Dow and Werlang showing that ambiguity can be an explanation for bid-ask spreads in partial equilibrium portfolio choice problems. Pennesi unifies and generalizes these results by considering a general class of recursive ambiguity averse preferences. He shows that asset prices are characterized by Euler inequalities, generated in each period by a set of probabilities relevant for that period. This set can be identified in the particular cases that have been studied in the literature, including maxmin, variational, and smooth ambiguity models, and in these cases it corresponds to a natural set of measures characterizing ambiguity in these functional forms. The general results illustrate that ambiguity can be understood as enriching the standard Lucas asset pricing formula through an additional stochastic discount factor, which can again be calculated in all of the standard cases. An important implication of this result is that price indeterminacy holds in general if and only if this set is not a singleton, thus if and only if there is sufficient nonsmoothness in the value function. This result suggests that price indeterminacy is not a consequence of ambiguity aversion alone, but instead is generated by the combination of ambiguity aversion and particular functional forms to represent it.

The idea of robustness to misspecification of a model of risk has been prominent in research on risk measurement for over two decades. The paper "Robust Return Risk Measures" by F. Bellini, R.J. Laeven and E. Rosazza Gianin is a contribution to this line of research. It provides foundations for a class of measures of return risk (or relative risk) called Orlicz risk measures, with and without consideration of robustness. These measures are based on Orlicz norms on the space of returns. The authors show that Orlicz risk measures of return risk are in one-to-one relationship with utility-based measures of shortfall risk of payoffs. The paper provides an axiomatization of Orlicz measures. Further, it introduces robust Orlicz measures which are based on multiple probabilistic models of risk rather than a single model.

Standard theory of portfolio selection postulates a single model for predicting returns on all assets. Reliance on a single model of returns is fraught with difficulties, in particular, when the number of assets is large. The paper "Combining different models" by Leonard Ch. Rogers proposes a method of combining different models for predicting returns of overlapping subsets of assets into one prediction for all assets. The method involves Bayesian averaging of different models on suitably selected subsets of assets, and using an entropy criterion to combine those averaged models into a single one. The paper shows that the method gives rise to a unique prediction and provides a solution in the case of multivariate Gaussian distribution.

The six papers of this issue reflect the wide range of topics that we discussed during the time at the Center for Interdisciplinary Research in 2015. We hope that you will find the papers of this issue as enlightening as we do-bon appétit!

Frank Riedel, Chris Shannon, and Jan Werner 